\title{
Féminin, féminité et diversité dans les albums Agrippine de Claire Bretécher depuis 1995
}

\author{
Adeline Caute \\ Université McGill \& \\ Collège Dawson
}

Dans les huit albums qui composent la série Agrippine de Claire Bretécher, le féminin domine. Outre le fait que c'est un prénom féminin qui orne les couvertures des livres, l'héroïne y est toujours représentée au premier plan. Dans deux albums (Agrippine [1988] et Allergies [2004]), elle est seule sur l'illustration de la couverture. Quand elle partage la couverture avec un personnage, l'autre est en retrait : c'est le cas de son arrière-grand-mère (dans Agrippine et l'ancêtre [1998] et Agrippine déconfite [2009]) qui fait la moitié de sa taille, et de son prétendant, Modern Mesclun, (Les combats d'Agrippine [1993]), qui est plus difficilement identifiable que sa comparse, car il a la tête renversée en arrière. Sur les couvertures des trois derniers albums, elle partage l'illustration avec plus d'une personne, mais elle occupe symboliquement et spatialement une plus grande importance que les autres, en se détachant devant un groupe de gens peu individualisés (Agrippine et les inclus [1995] et Agrippine et la secte à Raymonde [2001]), ou en étant au premier plan alors que les autres, en l'occurrence un couple, sont au second plan (Agrippine prend vapeur [1991]).
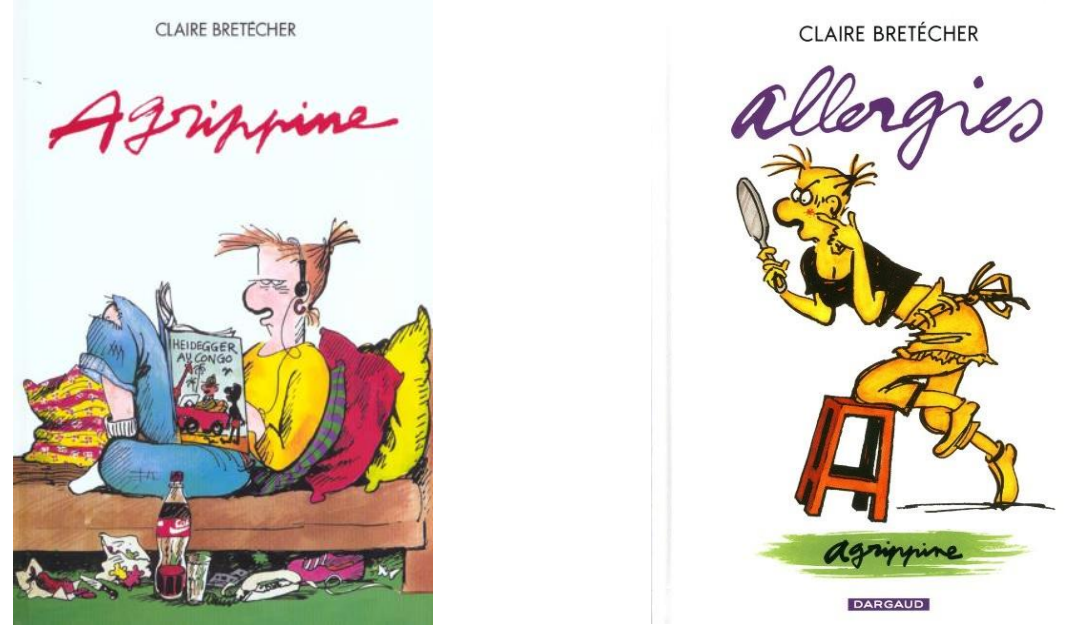

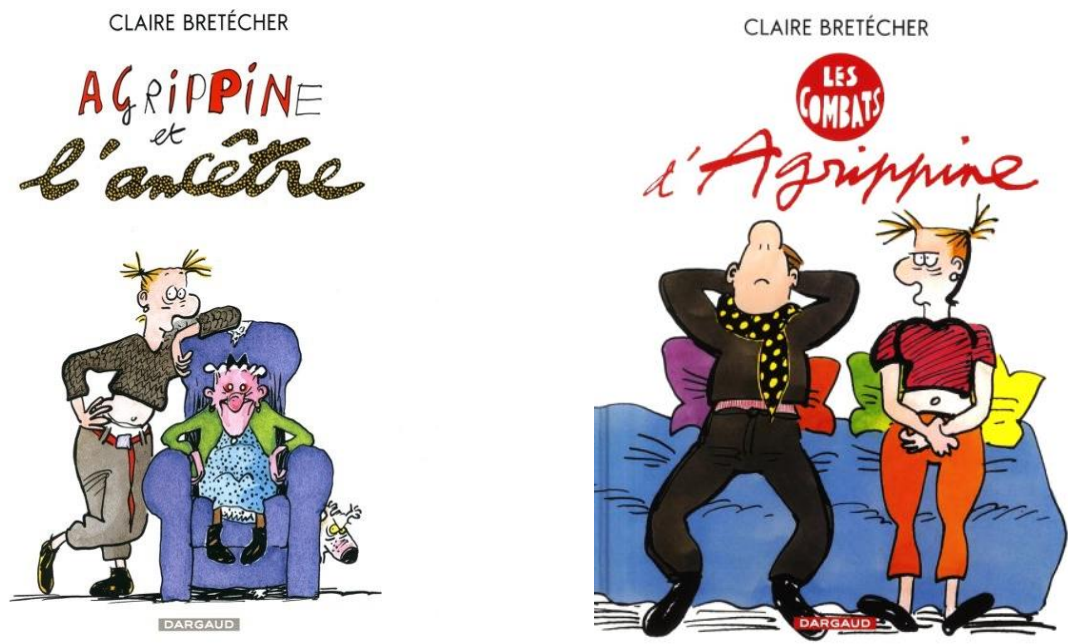

CLAIRE BRETÉCHER
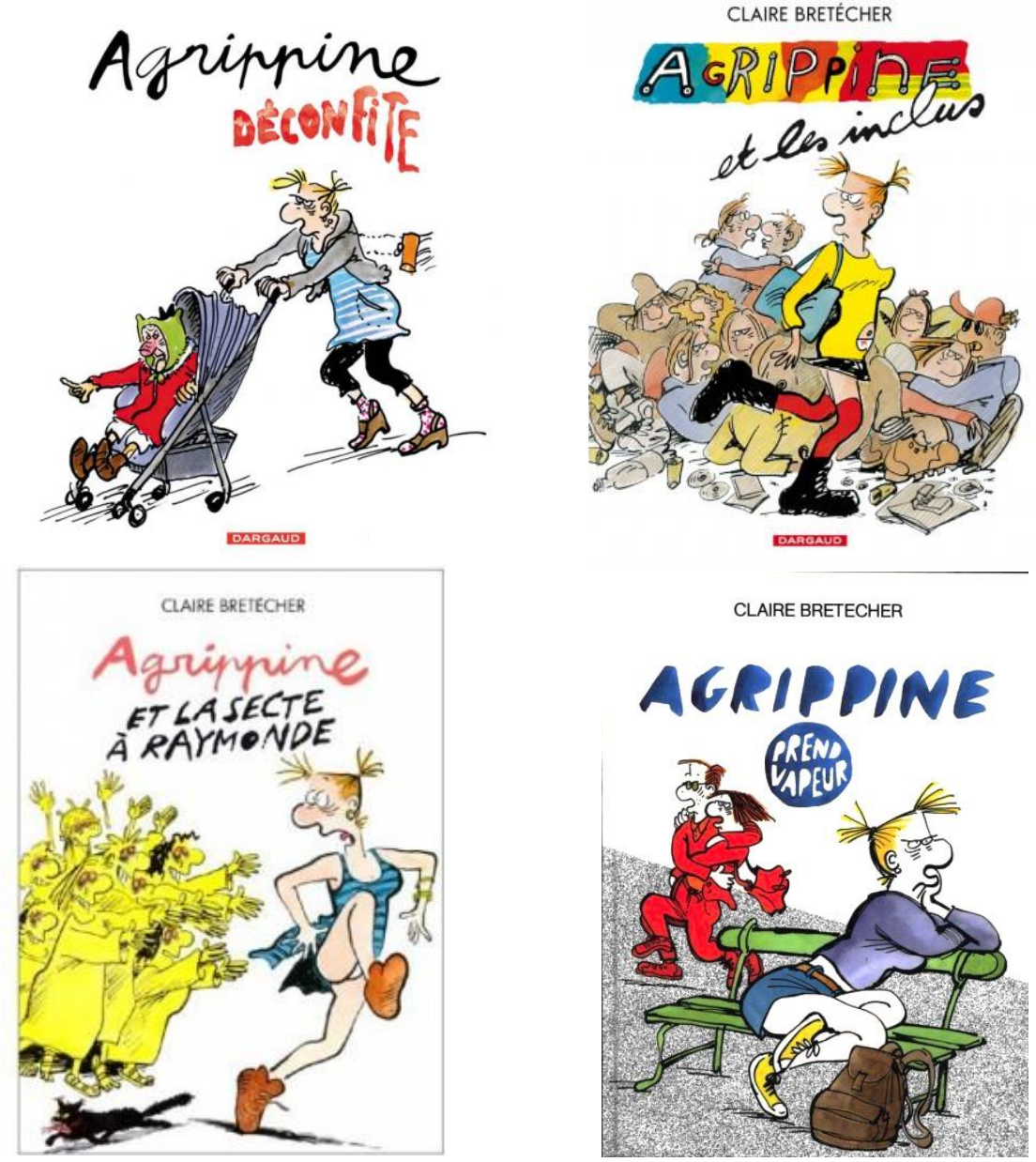

CLAIRE BRETECHER

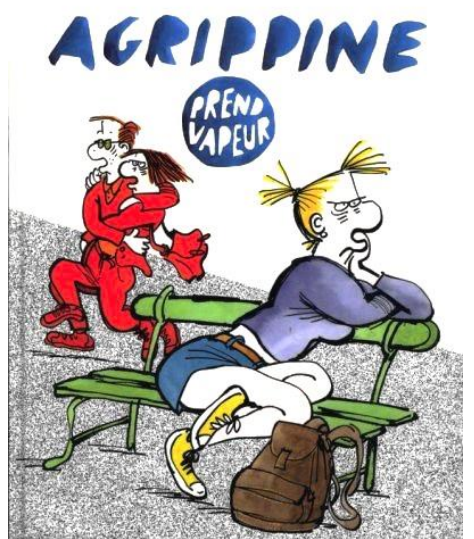


Toutefois, la surreprésentation proportionnelle du féminin n'est pas confinée aux couvertures et va plus loin que la préséance symbolique d'Agrippine. Si l'héroïne est de sexe féminin, c'est également le cas de la majorité des autres personnages. Au sein de la famille nucléaire d'Agrippine, masculin et féminin sont à égalité, puisque face à l'adolescente et à sa mère, Poule, se trouvent Biron, le frère d'Agrippine, et Merlan, son père, mais ce n'est pas le cas de sa famille élargie. En plus de Zonzon, l'arrière-grand-mère d'Agrippine qui la côtoie sur les couvertures, la grand-mère de l'adolescente (dite Mamie ou Ninifle) est aussi souvent associée aux aventures de la jeune fille, alors qu'aucun grand-père ou arrière-grand-père ne l'est. Les derniers membres au tableau de la vie familiale d'Agrippine sont Jean-Million, le frère de sa mère, et Candida, la femme de ménage, cette dernière étant largement plus présente que le premier. Le cercle social d'Agrippine est également plus féminin que masculin, puisque ses fréquentations principales sont ses amies Bergère Leprince, Psychée Chia et Moonlight Mollard, auxquelles s'ajoute parfois Rouge-Gorge de Cossé-Balzac. Agrippine a des amoureux ou des prétendants comme Morose Le Hachis, Modern Mesclun, Persil Wagonnet ou Canaan Linchbage, mais ils sont généralement moins importants que leurs consœurs.

Si l'univers d'Agrippine est marqué par une représentation importante du féminin, il se caractérise aussi par une certaine diversité. Nombreux sont les personnages dont le nom de famille a une consonance étrangère, comme Muflée Madredios, Rusti Sofistic ou Cornélius Cornedblum. Des personnages aux origines ethniques différentes sont également représentés en petit nombre, comme la policière noire qui procède à l'arrestation finale dans La secte à Raymonde. La diversité sociale, en revanche, est moins présente dans les albums, puisque Candida est la seule protagoniste récurrente à ne pas être issue d'un milieu bourgeois ou « bobo ».

Toutefois, constater la présence absolue ou relative du féminin ou de la diversité dans une œuvre donnée ne permet pas de déterminer les modalités de cette représentation. Dans les pages qui suivent, je vais m'intéresser aux choix de Bretécher et à leur évolution concernant la représentation et le positionnement des minorités visibles et invisibles (personnages féminins, de couleur, homosexuels, personnes âgées, ouvriers, etc.) dans les cinq albums publiés par Bretécher depuis 1995, soit Agrippine et les inclus, Agrippine et l'ancêtre, La secte à Raymonde, Allergies et Agrippine déconfite.

Sacrée en 1976 «meilleur sociologue français de l'année » (sic) par Roland Barthes qui saluait la profondeur des fresques de ses Frustrés paraissant alors dans Le Nouvel Observateur (Le Saux, n.p.), Claire Bretécher a signé trente-cinq 
albums de bandes dessinées depuis ses débuts au sein de l'équipe du journal Pilote en 1969. Dans une entrevue de 2009, elle affirme que l'idée d'Agrippine lui est venue « d'une espèce d'admiration générale pour les ados ». Elle poursuit :

Quoi qu'ils fassent, même si c'était des conneries, leurs parents les trouvaient géniaux. «Machinette a fait ça, c'est formidable, non ?» Tout était ainsi. Évidemment, quand on arrive à la came, à la prostitution et au hold-up, c'est moins sympa! Mais il y avait un regard à la fois tragique, admiratif et envieux sur ces jeunes. C'est de cette ambiance qu'est née Agrippine. [...] Je suis quand même très dépendante de l'actualité l'actualité des mœurs, parce que l'actualité politique ne m’intéresse pas du tout (Festraëts).

En effet, le lecteur de Bretécher remarquera l'évidente évolution des idiosyncrasies de l'adolescente, que ce soit en termes d'habillement ou de langage. Pour ne citer qu'un exemple, pensons à l'adverbe "grave » utilisé par Agrippine dans la phrase "wé grave», par laquelle elle manifeste son assentiment dans La secte à Raymonde ${ }^{1}$. Selon Jean-François Dortier, qui s'est intéressé au "parler jeune », le mot " peut signifier beaucoup, très ('Putain, tu me prends grave la tête?), mais peut aussi s'employer pour désigner une personne étrange ou bizarre, plutôt 'zarbi' ('Il est grave?) » (Dortier) et, en 2001, année de publication de l'album, son acception dans le sens où l'entend Agrippine est encore récente.

Toutefois, quand elle est interrogée sur la nature de sa motivation à composer les aventures d'Agrippine, Bretécher repousse l'idée que son œuvre s'apparente au sociologique ${ }^{2}$ : «Mon dessin relève moins du sens de l'observation que du sentiment d'appartenir à un groupe social. Je parle toujours plus ou moins de moi-même, et mes personnages se moquent beaucoup de mes propres travers. Il m'arrive d'avoir des réactions que je crois personnelles, avant de me rendre compte que tout le monde a les mêmes. [...] Mais les idées sont dans l'air. Il faut savoir les attraper»(Festraëts n.p.). Certains aspects de son œuvre, pourtant, l'intéressent particulièrement, comme ses personnages féminins ${ }^{3}$. En entrevue, Bretécher a souvent été interrogée sur eux, ainsi que son

1 C'est dans son environnement immédiat que Brétecher étudie ces idiosyncrasies générationnelles : «Quand mon fils avait 15 ou 16 ans, je l'écoutais parler au téléphone. Les enfants pratiquent plusieurs langues, en tout cas au moins deux. La plupart du temps, c'était nul, mais, parfois, je tombais sur une vraie perle. Maintenant, j'invente. Ce qui est drôle, c'est qu'aujourd'hui les ados se mettent à parler l'argot d'autrefois. Bon, ils ne le savent pas, mais cela ne fait rien » (Festraëts)

2 «- Roland Barthes a dit un jour de vous que vous étiez « le meilleur sociologue de France». Qu'en dites-vous?

- C'est vraiment n'importe quoi. » (Festraëts).

3 Selon Bretécher, «La grand-mère m'agace, l'oncle est un vrai con, les parents sont plutôt sympas et j'aime bien l'arrière-grand-mère. Mais Agrippine n'est pas très intéressante et son petit frère m'indiffère complètement » (Festraëts). 
rapport au féminisme. Alors qu'elle exprime une affection pour certaines femmes qu'elle prend plaisir à représenter dans ses livres («J'aime bien les filles qui bossent et qui sont un peu marrantes »), elle parle des hommes en général avec distance : «Je ne sais pas faire fonctionner les personnages masculins. Je ne comprends pas leur mécanique. Tout le monde me dit qu'il n'y a rien à comprendre, mais c'est faux. Il y a quand même des constantes par sexe et par mode de vie » (Festraëts). À la question «Vous sentez-vous féministe ? », en 2009, Bretécher répond au passé : «C'était ma tendance, mais l'aspect militant m’a toujours dégoûtée. Jeune, j'étais sur mon pré carré, fallait pas qu'on m’emmerde. [...] Maintenant, je m’en fous. Je ne me sens plus concernée. Ce n'est plus mon problème. Que les autres se démerdent !» (Festraëts) Toutefois, dans un tchat de la même année, après avoir réitéré son désintérêt pour le militantisme sous toutes ses formes, elle nuance un peu son propos : « quand on est une femme et qu'on est sainement égoïste, on est féministe ». Et elle conclut : «je m'élève violemment qu'il y ait [sic] le moindre sexisme dans Agrippine» (Festraëts). Autrement dit, si, dans les albums d'Agrippine, et notamment ceux parus depuis 1995 , le féminin et la féminité ${ }^{4}$ sont des thèmes centraux, le prosélytisme n’intéresse aucunement Bretécher.

Souvent associé à la féminité, le thème de l'apparence est constamment abordé dans l'univers d'Agrippine, de manière aussi bien implicite qu'explicite. Implicite, il l'est par le simple fait que tous les personnages changent de tenues plusieurs fois par histoire, sinon complètement, du moins en partie, y compris les personnages secondaires. Ainsi, Raymonde, qui s’identifie comme « la sœur de Dieu» dans l'album La secte à Raymonde, troque l'écharpe violette de sa première apparition (7) contre une étole dorée à l'aspect liturgique brodée de ses initiales lors de sa deuxième et dernière apparition (49). Traditionnellement, les auteur(e)s de livres d'images ou de bandes dessinées ont souvent prêté une tenue unique à leurs personnages, comme Bécassine ou Tintin. À ce sujet, Sylvain Rhéault, auteur d'un article intitulé «Les choix des créatrices de B.D. » écrit que «si cela facilite la reconnaissance [...], cela bloque, en revanche, toute possibilité d'évolution identitaire d'un épisode à l'autre» (91). Fait intéressant, pour Agrippine, l'absence de cet « uniforme » ne traduit pas d'évolution identitaire de la protagoniste, qui reste éternellement adolescente et dont les préoccupations ne changent que dans une mesure mineure d'un album à l'autre, mais exprime son inscription dans un contexte socioculturel, c'est-à-dire dans une époque, à

\footnotetext{
4 Précisons que dans le présent article, j'utiliserai ces deux mots comme non interchangeables, au sens où les ont définis des auteures féministes comme les psychologues Monique CournutJanin ou Jacqueline Godfrind, respectivement auteure en 1998 de Féminin et féminité et de Comment la féminité vient aux femmes (2001). Par « féminin », j'entendrai le fait d'être une femme, tandis que par « féminité », j’entendrai la manière dont on apprend culturellement à devenir une femme.
} 
l'instar de son langage. Explicite, le thème de l'apparence l'est également lorsque l'un des personnages parle de vêtements, de maquillage ou encore de coiffure. Par exemple, Agrippine déconfite ouvre et ferme sur la question des vêtements : les trois premières pages de l'album portent sur les chaussures qu'Agrippine désire se faire offrir par sa grand-mère, mais que cette dernière achète finalement pour elle-même, quand elle découvre que le magasin n'a plus la pointure d'Agrippine, mais a encore la sienne. La dispute que provoque cette situation conduit Agrippine à souhaiter à voix haute la mort de sa grand-mère, laquelle disparaît peu de temps après. Désorientée et rongée par la culpabilité, l'adolescente rend hommage à sa grand-mère en se présentant à sa cérémonie d'adieux en portant le costume qu'elle a choisi pour l'anniversaire d'une amie dont le thème est « la tenue que votre Grand-Mère aurait adoré porter». La cérémonie est interrompue par l'arrivée de la grand-mère qui porte les chaussures ayant déclenché le drame, mais Agrippine, soulagée, ignore la provocation. Autrement dit, dans l'album, l'apparence n'est pas seulement un thème important, c'est véritablement un enjeu capital des relations entre personnages.

Par ailleurs, l'intensité des rivalités entre Agrippine et sa grand-mère dit clairement que les vêtements jouent un rôle de premier ordre dans la représentation et l'autoreprésentation identitaires des personnages. Récemment, des chercheuses comme Dani Cavallaro et Alexandra Warwick se sont intéressées à la symbolique du vêtement dans une culture où le corps féminin est presque toujours associé au désir, en tant qu'objet d'un regard masculin. Ainsi écrivent-elles :

Dress, as a pervasive vehicle for the fabrication of culturally viable subjects, is in a position to increase our sensitivity to the gap between putatively natural body and its represented, artificial counterparts ; to the body's translation by means of mechanisms of rhetorical displacement ; and to structures of visuality between the observer and the representation. (Cavallaro et Warwick 4).

C'est précisément ce que révèle l'obsession d'Agrippine pour son corps, une obsession qui s'étend d'ailleurs à ses cheveux, comme le lecteur le découvre dans la planche intitulée «Tendance » dans Allergies. Chez Bretécher, le corps dit «naturel ${ }^{5}$ est transformé par les vêtements et la coiffure, et l'espace qui le sépare du nouveau corps habillé et fardé est à la mesure de la rage d'Agrippine qui se voit voler ses chaussures par sa grand-mère. Les vêtements et la coiffure d'Agrippine la font devenir non pas qui elle est, mais qui elle veut être et comment elle veut être perçue. En portant les «boots» de l'adolescente, la grand-mère lui prend son identité d'une manière qui semble non pas seulement superficielle, mais essentielle, car le vêtement a le pouvoir de transfigurer et de

\footnotetext{
${ }^{5}$ On notera qu'Agrippine apparaît souvent nue dans les albums, ce qui fait sortir les artifices de ses tenues généralement ridicules.
} 
«traduire », pour reprendre le mot de Cavallaro et Warwick, l'être, et, ce faisant, a le pouvoir de déclencher ou d'arrêter le désir de l'autre. À ce titre, il est intéressant de noter que les premières vignettes d'Agrippine déconfite ne présentent pas le mot «chaussure » ou autre expression équivalente : ce sont des pronoms objets et sujets sans antécédents qui les désignent («les», "elle») avant qu'Agrippine passe sa commande auprès de la vendeuse et, pour cela, utilise un substantif ( «je voudrais essayer ces boots en $42 »)$. Cette absence de référent direct crée un mystère autour de l'achat pour le lecteur, tandis que le recours à un mot anglais ("boots», que prolonge phonétiquement le nom de la boutique, "CHOUZ»), place les chaussures du côté de l'exotique et du «cool». En d'autres termes, le mode de présentation de ces dernières montre qu'elles sont bien plus que de simples objets et touchent à l'identitaire dans l'univers d'Agrippine.

Le choix des chaussures semble particulièrement symbolique dans ce contexte. Selon la psychanalyste Marie-Louise Pierson interrogée par la chroniqueuse Julie Champagne, c'est peut-être le vêtement le plus étroitement lié à notre conception de notre identité :

Notre pied revêt une symbolique particulière. D'un point de vue psychanalytique, le pied qui se glisse dans la chaussure est une évocation du coït, le pied étant le sexe masculin et la chaussure étant le sexe féminin. De plus, non seulement notre pied soutient notre corps, mais il influence aussi notre démarche toute entière. Nos chaussures changent donc la façon dont nous attaquons le sol et, par extension, la façon dont nous avançons dans la vie. [...] Il est possible de modifier complètement la perception de notre corps en fonction de la chaussure qu'on porte (Champagne).

Cette notion de la perception de son corps et de son identité explique l'ardeur du ressentiment d'Agrippine vis-à-vis de sa grand-mère. Le motif des chaussures objet de désir combiné au thème du rejet est également présent dans une série de deux planches de l'album Allergies. Dans la première planche, "Assistance ", Bergère, l'amie d'Agrippine, a pris en charge l'acquisition d'une paire de chaussons pour sa voisine âgée et grabataire, mais elle les a pris marron alors que la voisine les voulait gris. Elle raconte l'anecdote à Agrippine qui comprend le mécontentement de la voisine : « des chaussons marron!» s'exclame-t-elle avec incrédulité, avant de répéter, main en travers des yeux, « marron... pitié ! », puis, dans la vignette suivante, elle se contente de répéter « marron». Plus loin, elle précise sa pensée: "quand j'aurai 90 ans même si le pape en personne m'apporte des chaussons marron c'est 2 claques direct». Quand Bergère propose à Agrippine d'offrir les chaussons à son arrière-grand-mère, elle ne rencontre qu'indignation : " offre tes putain de chaussons marron à mon arrièregrand-mère et je te ravage la crête ». Dans la planche suivante, «Dérapages », Zonzon a reçu anonymement des chaussons marron et Agrippine tente de la convaincre de les échanger contre des gris, d'autant plus qu'ils glissent. 
Toutefois, Zonzon préfère garder les chaussons marron et changer le sol de sa chambre à la résidence. En effet, elle demande à son arrière-petite-fille de lui acheter « 25 mètres carrés de lino ». De ces deux planches ressort la très forte polarisation des deux femmes envers les chaussons, d'une part, l'horreur d'Agrippine et, d'autre part, l'ardeur de l'attachement de son arrière-grand-mère. Le message est clair : dans l'univers d'Agrippine, choisir ce qu'on enfile à ses pieds est un geste lourd de sens.

Si la féminité est traditionnellement associée à la représentation du corps, chez Bretécher, les hommes aussi ont un rapport identitaire complexe aux habits. Les camarades de classe d'Agrippine se changent fréquemment et les personnages secondaires ne sont pas en reste. À deux occasions, le rapport des personnages de sexe masculin à leurs vêtements révèle un conflit identitaire intérieur qui s'exprime de manière générique. À la fin de La secte à Raymonde, deux policiers font une descente dans la salle où Raymonde harangue ses ouailles, un homme et une femme (53-54). Tous deux portent des vêtements de civil très voyants, mais l'homme, Frimo, s'intéresse beaucoup plus à l'esthétique des lieux qu'aux crimes que commet Raymonde. Ainsi exprime-t-il son enthousiasme pour la salle au complet ("c'est crognon ici»), l'état des murs («oh, il est choupinou ce camaïeu de salpêtre »), la lumière («il est mimi l'éclairage!»), à nouveau l'état des murs (" oh... chatounet ce décrépi »), et, pour finir, la tenue d'Agrippine ("il est beau votre boléro... un collector vintage ?»). Sa collègue le ramène à l'ordre : " putain Frimo sois un peu à ce que tu fais... on le saura que tu voulais faire design et deco (sic) d'intérieur» (54). Le sérieux de la policière, peu intéressée par l'apparence, rend encore plus visible la distraction de Frimo, dandy efféminé (il se tient très cambré et porte des chaussures à talons) et affecté, mais Bretécher a poussé plus loin l'effet de contraste en choisissant de représenter la partenaire de Frimo non seulement sous les traits d'une femme, mais d'une femme noire aux cheveux crépus portant des créoles surdimensionnées. Dans un milieu, celui de la police, traditionnellement dominé par les hommes blancs et l'hétéronormativité, le personnage de Frimo exprime sa différence et ses ambitions contrariées. Dans Agrippine déconfite, Biron vit une situation similaire. Tout d'un coup, il se met à emprunter les bijoux de sa sœur et s'habille avec des vêtements féminins. Au début de l'album, il porte « le serretête à noeunoeud » d'Agrippine (11), puis, à la page suivante, il arbore une robe à col Claudine. Peu après, il annonce à sa famille : «je m’appelle Patata je suis danseuse au Moulin-Rouge » en chaussures à talon et soutien-gorge rose. Plus loin, il est représenté avec des boucles d'oreilles, et, pour la cérémonie d'adieux de sa grand-mère, il a enfilé ce que son père décrit comme un « ridicule voile de veuve » (47). Comme l'indique l'adjectif « ridicule », Merlan trouve à redire à sa tenue. En revanche, ni Agrippine ni sa mère ne semblent déroutées ou même surprises par les nouveaux choix vestimentaires du petit garçon. Ainsi, lorsque 
Merlan s'exclame "puis-je demander pourquoi mon fils porte une robe?», Agrippine répond «c'est rien papa, c'est juste qu'il est un peu transsexuel en ce moment ». Cela n'empêche pas Merlan d'enjoindre sévèrement à son fils « tu vas m'enlever cette robe et plus vite que ça » puis « eh bien enlève aussi la poudre et le porte-jarretelles » (12). Dans cette dernière tirade du père de famille, le recours à un pronom complément indirect ( me », contracté dans l'expression «m'enlever») montre non seulement que Merlan prend très au sérieux le travestissement de son fils, mais qu'il en fait une affaire personnelle.

Pour ce qui est de la représentation de la diversité ethnique, les derniers albums de Bretécher lui accordent une place largement plus importante que les premiers. Alors que dans le premier et le troisième albums, Agrippine et Les combats d'Agrippine, la totalité des personnages sont blancs et que le deuxième, Agrippine prend vapeur ne présente qu'un personnage non-blanc, qui s'avère également étranger puisqu'il parle anglais à Rouge-Gorge (37), à partir d'Agrippine et les inclus, les personnages sont plus diversifiés. Dans l'album de 1995, un personnage noir apparaît à deux occasions dans des scènes de groupes d'amis d'Agrippine (13 et 38). Dans l'album suivant, Agrippine et l'ancêtre, un personnage à la peau foncée accompagne Bergère (36), et Biron a un camarade de classe noir et un autre, arabe (42-43). On notera également que l'album présente une protagoniste bretonne, Gwinamouc'h, alors que les prénoms et noms des personnages ne laissaient pas supposer leurs origines régionales jusqu'alors. Dans Agrippine et la secte à Raymonde, en plus de la policière noire déjà mentionnée (53-54), deux personnages asiatiques, Lama Gaga et Lama Dodo (23-25), et Candida (24-25), que Bretécher avait déjà dessinée dans Docteur $V$ entouse, bobologue et dans Le destin de Monique, font leur apparition. Toujours dans Agrippine et l'ancêtre, un client et une employée de la Fnac ont la peau foncée (32), ainsi qu'un camarade de classe d'Agrippine (42) et une personne de la foule qui écoute Raymonde (50). Dans Allergies, une cliente du magasin où travaille Agrippine et Guirlande, l'amie d'un ami de l'adolescente, ont la peau foncée (dans «Clientizing » et «Félina »), et Agrippine a une camarade de classe noire («Rebelles »); de plus, deux personnages secondaires qui font la queue pour le même casting qu'Agrippine sont noires («Le langage du corps »). Enfin, dans Agrippine déconfite, un personnage peu défini a la peau noire (6) et deux piétons ont la peau foncée (18-19), de même que Jean-Maurice, l'aumônier de la maison de retraite de Zonzon (25-26), quatre employés de l'équipe de TVMotion (29 et suivantes), Andante Cohn Motto, ami d'Agrippine (37-39) et un membre du binôme envoyé par TVMotion pour suivre Jean-Mi chez Agrippine. Bref, de toute évidence, le monde d'Agrippine a changé et reflète de plus en plus la diversité de la société parisienne. Socialement, en revanche, la majorité des personnages appartiennent aux bourgeois-bohèmes rendus célèbres par 
Bretécher dans ses Frustrés ${ }^{6}$, à quelques exceptions près, dont, bien entendu, Candida, mais aussi un vieux délinquant, anciennement proxénète, dans Agrippine et les inclus (27) ou un sans-abri dans la planche « Rebelles » d'Allergies.

Globalement, l'univers d'Agrippine n'en est pas moins de plus en plus inclusif, pour reprendre le mot du titre de l'album de 1995. Cela est peut-être lié au fait que le monde d'Agrippine s'élargit de plus en plus, en dépassant les murs de l'appartement de ses parents et de son lycée. Parallèlement, les situations dans lesquelles sont représentées les minorités visibles et invisibles les font gagner en agentivité. C'est particulièrement le cas de Poule, la mère d'Agrippine, qui est toujours habillée en tenue de ville, ce qui la désigne comme une femme professionnellement active. Elle est énergique, efficace et sûre d'elle. Il est à noter que ces caractéristiques ressortent d'autant plus que, par contraste, ses deux alter ego - son mari et son frère - semblent passifs, rêveurs ou mous. Ainsi Merlan est-il souvent représenté en sous-vêtements ou nu. Dans ses quatre apparitions dans Agrippine et la secte à Raymonde, il est plus souvent en pyjama ou en peignoir qu'habillé. De plus, au début de l'album, la première fois que le lecteur le voit, il vient de sortir du lit (il n'est pas rasé et porte un pyjama et un peignoir) et y retourne très vite. Sa posture passe de courbé à allongé, tandis que Poule est debout, prête à sortir, un café à la main. Le second alter ego de Poule, son frère Jean-Mi, va d'échec professionnel en escroquerie ratée. Ironie du sort, malgré ses protestations, il passe son temps à demander de l'argent à sa mère ( ARRÊTE MAMAN! tu crois toujours que je veux te taper, c'est énervant à la fin quoi [...]. Si tu avais 2 ou 3000 euros en cash ça me dépannerait », Agrippine déconfite, 22) alors que cette dernière s'est efforcée de l'aider à réussir en choisissant son prénom, Jean-Million : « je voulais qu'il réussisse dans la vie et j'estime qu'il faut donner aux enfants des prénoms qui les motivent». Lorsqu'Agrippine lui demande "alors pourquoi pas Jean-Milliard?», elle précise : «j’ai pensé million de dollars et puis il faut être raisonnable » ("Au nom du fils », Agrippine et les inclus, 47). Si le prénom de son fils fait donc explicitement référence au commerce international, celui de sa fille renvoie à un destin maternel, profondément ancré dans l'espace privé et intérieur. Pourtant, ce sont les femmes qui réussissent dans l'univers d'Agrippine. Certes, Merlan est un écrivain avec des relations importantes, puisqu'il parvient à obtenir sans difficulté le numéro de téléphone portable du ministre de « l'Éduc nat 》 (La secte à Raymonde, 12) et est bien loin des échecs de son beau-frère, mais Poule a une conférence téléphonique avec le ministre slovène (Agrippine déconfite, 7). La mère de Poule est riche grâce à son premier mari (qu'elle n’a pas «épousé pour ses idées mais pour sa fortune ", Agrippine et l'ancêtre, 11), sa grand-mère, Zonzon,

\footnotetext{
${ }^{6}$ Et ce, même si le mot apparaît pour la première fois sous la plume de Maupassant dans BelAmi en 1885. Chez Bretécher, les « bobos » sont d'anciens soixante-huitards de gauche.
} 
est une championne de la bourse (Agrippine et l'ancêtre, 24) et la grand-mère de Zonzon était « couturière légère chez la comtesse OBKUK de KUK » (Agrippine et l'ancêtre, 26). De façon générale, dans les derniers albums d'Agrippine, les mères et la maternité sont omniprésentes, jusque dans des détails tels que le choix de l'aria qu'écoutent Poule et Merlan («La mamma morta de la Callas », Agrippine déconfite, 10) et la marque de la bière préférée de Jean-Mi («Mother Ale » « Specially pissed by Mums to pour down the gob of their moronic sons », Agrippine déconfite, 8). Chez Agrippine, la généalogie est matrilinéaire et les femmes, tous milieux et origines confondues, sont associées à la réussite et à une forme d'autorité. Comme mentionné précédemment, dans Agrippine et la secte à Raymonde, la policière est bien plus efficace que son collègue, Raymonde, la sœur de Dieu, est la cheffe incontestée de la secte à succès qui porte son nom, et, dans Agrippine déconfite, c'est auprès d'une femme noire, Hazelmia, décrite comme «boulot-boulot plus le talent », que Jean-Mi tente de vendre son projet de téléréalité (30-31). En outre, Jean-Mi laisse à sa sœur le soin de rencontrer le notaire suite au (faux) décès de leur mère - et en profite pour poser des micros dans son appartement (40). Dans ses relations amoureuses, malgré un désir évident de séduire, Agrippine mène le jeu. Ainsi, dans Allergies, une illustration représente Agrippine nue, à califourchon et à l'envers sur un garçon tout jaune menotté au lit et elle appelle quelqu'un, dont le lecteur suppose que c'est une amie, pour demander « c'est moi... j'ai un problème : à ma place est-ce que tu mettrais une capote à un vibro ? NON NE ME PASSE PAS TA MÈRE ». À l'inverse, lorsqu'un homme invoque le genre d'Agrippine avec condescendance, les événements lui donnent tort et il paraît ridicule : ainsi, dans Agrippine et la secte à Raymonde, Rusti Sofistic propose à Agrippine de lui vendre son scooter qui marche à la prière et lui lance « je te passe les détails trop techniques pour une femme »; toutefois, quand il tente une démonstration en s'agenouillant devant le scooter et en priant Raymonde, le scooter ne démarre pas (29). Mais c'est peut-être Candida qui incarne le mieux cette tendance d'un féminin fort. Dans Agrippine déconfite, elle annonce au téléphone à un-e ami-e qu'elle pense à quitter la maison d'Agrippine à cause de l' «ambianche époubantavle [sic] ». Effrayée à l'idée d'être licenciée et de devoir " retourner aux proud'hommes [sic]», elle n'en tient pas moins tête à Jean-Mi à qui elle reproche de ne pas être chez le notaire avec Poule (" parfois j'oublie de vous demander votre avis Candida », lui répondil (40) avant de réagir assez grossièrement à une de ses plaisanteries : «HA HA ekchellent ! bous abez pris des cours dou choir d'humour en Corée dou Norde? [sic]» (40). Énergique, directe, loyale, efficace en affaires, Candida est une non seulement une femme, mais une femme corpulente «ichou[e] de la diberchité » à l'instar de «[s]es fianchés [sic]» (40) et constitue un des personnages les plus positifs des albums. 
En conclusion, si Bretécher se refuse au prosélytisme et au militantisme, il n'en reste pas moins qu'au cours des vingt-cinq dernières années, l'univers de ses albums s'est enrichi en accueillant des personnages de plus en plus divers et en offrant une place de choix à des protagonistes toujours plus variés. Derrière une obsession pour leur apparence qui les poursuit jusqu'à la maison de retraite, les femmes de Bretécher sont, à l'image des minorités visibles et invisibles des albums d'Agrippine, peintes comme des personnages intelligents, forts, ouverts à la différence, pleins d'humour et d'humanité. 


\section{Bibliographie}

Bretécher, Claire. Agrippine. Paris : Claire Brétecher (éditrice), 1988.

Bretécher, Claire. Agrippine prend vapeur. Paris : Claire Brétecher (éditrice), 1991.

Bretécher, Claire. Les Combats d'Agrippine. Paris : Claire Brétecher (éditrice), 1993.

Bretécher, Claire. Agrippine et les inclus. Paris : Claire Brétecher (éditrice), 1995.

Bretécher, Claire. Agrippine et l'ancêtre. Paris : Hyphen, 1998.

Bretécher, Claire. Agrippine et la secte à Raymonde. Paris : Hyphen, 2001.

Bretécher, Claire. Allergies. Paris : Hyphen, 2004.

Bretécher, Claire. Agrippine déconfite. Paris : Dargaud, 2009.

Bretécher, Claire. "Il n'y a pas le moindre sexisme dans Agrippine », tchat, Libération, Web. 10 janvier 2016.

Cavallaro, Dani et Alexandra WARWICK. Fashioning the Frame: Boundaries, Dress and The Body. Oxford : Berg, 1998.

Champagne, Julie. "Ce que vos chaussures disent de vous », Moi \& Cie. Web. 19 jan. 2016.

Cournut-Janin, Monique. Féminin et féminité. Paris: Presses Universitaires de France, 1998.

Dortier, Jean-François. " 'Tu flippes ta race, bâtard!' Sur le langage des cités », Sciences humaines. Web. 16 jan. 2016.

Festraëts, Marion. «Claire Bretécher: 'Je suis raisonnablement misanthrope', L'Express. 19 mars 2009. Web. 16 jan. 2016.

Godefrind, Jacqueline. Comment la féminité vient aux femmes. Paris: Presses Universitaires de France, 2001.

Honorez, Amélie. La représentation du genre féminin dans la bande dessinée québécoise. Mémoire de maîtrise déposé à l'Université du Québec à TroisRivières, 2012.

Le Saux, Laurence. "Les Frustrés de Bretécher: bobos des années 1970 », Télérama, 17 novembre 2015. Web. 10 jan. 2016.

Pilloy, Annie. Les Compagnes des héros de B.D. Des femmes et des bulles. Paris: L'Harmattan, 1994.

Pilloy, Annie. "Les stéréotypes féminins dans la BD pour enfants et adolescents », Regards sociologiques, $\mathrm{n}^{\circ}$ 9-10 (1995): 39-45.

Rhéault, Sylvain. «Les choix des créatrices de B.D. », Voix plurielles 9-2 (2012): 91-104.

Sadoul, Jacques. L'enfer des bulles : 20 ans après. Paris : Albin Michel, 1990. 